positive and were administered daily oral doses of MCF extract $(150 \mathrm{mg} / \mathrm{kg})$ daily for 21 days and subsequently injected i.p. with $\mathrm{CCl}_{4}(50 \% \mathrm{v} / \mathrm{v}$ in olive oil; $1 \mathrm{ml} / \mathrm{kg})$ on the 22nd day. $\mathrm{CCl}_{4}$-induced damages were assessed through liver function markers viz.; alkaline phosphatase, alanine transaminase, aspartate transaminase, and lactate dehydrogenase. Changes in lipid profile were checked by measuring serum total cholesterol, triglycerides, high-density lipoproteins and low-density lipoproteins. Antioxidant status was checked by the activities of antioxidant enzymes (superoxide dismutase, glutathione peroxidise ), malondialdehyde (MDA)content. The histopathological changes were observed with Masson staining. Results Administration $\mathrm{CCl}_{4}$ induced an elevation of serum amino- and glutamyl transferases activities and an increased peroxidation, as well as a decrease of superoxide dismutase and glutathione peroxidise activities in the liver. Administration of $\mathrm{CCl}_{4}$ in rats caused a significant increase in liver function and lipid profile indicating hepatic damages which were restored by co-administration of MCF extract. Cellular and DNA damages in hepatic tissues were caused by $\mathrm{CCl}_{4}$ which shown clear hepatic fibrosis in addition to disturbing antioxidant enzyme level. Co-treatment with MCF extract regulated these markers of oxidative dysfunctions. MCF extracts enhance hepatic glutathione and may contribute to the antioxidant defense of the liver.

Conclusions It may be concluded that MCF extracts have the ability to reverse $\mathrm{CCl}_{4}$ induced hepatic damages. Momordica charantia fruit has been used to treat alcoholic liver disease, acute and chronic viral hepatitis and toxin-induced liver diseases.

\section{IDDF2019-ABS-0263 ANDROGEN RECEPTOR PROMOTES GASTRIC CARCINOGENESIS VIA UPREGULATING THE EXPRESSION OF CELL CYCLE-RELATED KINASE}

\begin{abstract}
${ }^{1}$ Xiaoxing Li*, ${ }^{1}$ Mengke Chen, ${ }^{1}$ Xiaoyi $\mathrm{Xu},{ }^{1}$ Ren Wang, ${ }^{2}$ Lixia $\mathrm{Xu},{ }^{2}$ Ning Zhang, ${ }^{3}$ Guijun Zhao. ${ }^{1}$ State Key Laboratory of Oncology in South China, Collaborative Innovation Center for Cancer Medicine, Sun Yat-Sen University Cancer Center, Guangzhou, Guangdong, China; '2Department of Gastroenterology, The First Affiliated Hospital, Sun Yatsen University, Guangzhou, Guangdong, China; ${ }^{3}$ Department of Gastroenterology and Hepatology, Inner Mongolia People's Hospital, Hohhot, Inner Mongolia Autonomous Region, China
\end{abstract}

\subsection{6/gutjnl-2019-IDDFabstracts. 104}

Background Gastric cancer (GC) is a leading global health problem. In most areas of the world, the incidence rate of GC in males was 1.5- to 3- fold higher than that in females. The androgen receptor (AR) is an independent adverse prognostic factor in patients with GC. However, the mechanism of AR regulating the progression of GC remains unclear. The aim of this study is to determine the effect of AR on the progression of GC and the mechanism behind these effects, which will provide novel ideas for the treatment of GC.

Methods The relative expression of AR were detected by semi-quantitative RT-PCR and real-time quantitative PCR. The functions of $\mathrm{AR}$ in GC were determined by colony formation experiment, transwell migration and invasion assay. The clinical data were obtained from the website cBioPortal and XENA database. The potential mechanism of AR in GC was searched by Gene Set Enrichment Analysis (GSEA) and DAVID. Chromatin immunoprecipitation (ChIP) and PCR were performed to explore the function of AR as a transcriptional factor. The xenograft mouse models in nude mice were used to verify the function of CCRK in vivo.

Results The expression of AR was upregulated in 6/8 GC cell lines. Compared to adjacent tissues, it's expression of GC was higher. Ectopic expression of AR promoted the colony-formation ability, migration and invasion of GC cells. In contrast, the knockdown of AR showed the opposite effects. Remarkably, we found that AR regulated the expression of cell cycle related kinase $(\mathrm{CCRK})$ through transcriptional regulation. The AR-CCRK axis promoted GC development through phosphorylation of GSK3 $\beta$ and $\beta$-catenin. Furthermore, TCGA data revealed that high expression of AR or CCRK was related with poor prognosis of GC patients. The prognosis of patients with concurrent high expression of AR and CCRK was significantly worse than that of patients with low expression of both AR and CCRK.

Conclusions The expression of CCRK is increased by AR in GC. Low expression levels of AR and CCRK are related to better prognosis in GC patients, suggesting they are also candidates as prognostic indicators in GC.

\begin{tabular}{|l|l}
\hline IDDF2019-ABS-0264 & HEPATIC CELL CYCLE-RELATED KINASE \\
& SHAPES A METASTATIC-PRONE LIVER \\
& MICROENVIRONMENT VIA CROSSTALK \\
& BETWEEN MYELOID-DERIVED SUPPRESSOR \\
& CELL AND NATURAL KILLER T CELL
\end{tabular}

Xuezhen Zeng ${ }^{*}$, Jingying Zhou, Zhewen Xiong, Hanyong Sun, Weiqin Yang, Wenshu Tang, Yu Feng, Alfred Cheng. The Chinese University of Hong Kong, Hong Kong

\subsection{6/gutjnl-2019-IDDFabstracts.105}

Background Metastasis is a prominent cause of cancer-related death governed by both cancer cell-intrinsic mechanisms and extrinsic microenvironment. Clinical observations demonstrated liver as a common metastatic site for various cancers, which may be due to its immune tolerant environment. Myeloidderived suppressor cell (MDSC) is a heterogeneous cell population of immature myeloid cells that contribute to the formation of a favorable metastatic environment partially via suppression of immune effector cells. However, the underlying mechanisms in liver tropism of tumor metastasis remain poorly understood. We have previously discovered that cell cycle-related kinase (CCRK) can promote primary hepatocellular carcinoma (HCC) development via MDSCs. Here we hypothesize that the accumulation of hepatic MDSCs induced by CCRK may contribute to the formation of a favorable metastatic liver microenvironment.

Methods We constructed a liver-specific CCRK inducible transgenic (TG) mouse model by a Cre/loxP system. Orthotopic and metastatic mouse models were used to investigate the role of CCRK in promoting tumor growth and metastasis. PMNMDSC was depleted by anti-Ly6G antibody. Cytokine, chemokine, and immune cells profiling were performed after sacrifice.

Results Induction of CCRK expression by tamoxifen injection could increase $\mathrm{CD} 11 \mathrm{~b}^{+} \mathrm{Gr}-1^{+} \mathrm{Ly} 6 \mathrm{G}^{+} \mathrm{Ly} 6 \mathrm{C}^{\text {low }}$ polymorphonuclear (PMN)-MDSCs liver accumulation specifically in male mice with upregulated Cxcl1 and Gcsf expression. Intrahepatic injection of a mouse hepatoma cell line Hep1-6 in male TG mice developed larger tumors compared to control and positively associated with increased PMN-MDSCs levels in liver. 